\title{
Distributed Service Restoration of Active Electrical Distribution Systems using ADMM
}

\author{
López, Juan Camilo ; Rider, Marcos J. ; Shen, Feifan; Wu, Qiuwei
}

Published in:

Proceedings of the 10th Conference on Innovative Smart Grid Technologies

Link to article, DOI:

10.1109/ISGT.2019.8791626

Publication date:

2020

Document Version

Peer reviewed version

Link back to DTU Orbit

Citation (APA):

López, J. C., Rider, M. J., Shen, F., \& Wu, Q. (2020). Distributed Service Restoration of Active Electrical Distribution Systems using ADMM. In Proceedings of the 10th Conference on Innovative Smart Grid Technologies IEEE. https://doi.org/10.1109/ISGT.2019.8791626

\section{General rights}

Copyright and moral rights for the publications made accessible in the public portal are retained by the authors and/or other copyright owners and it is a condition of accessing publications that users recognise and abide by the legal requirements associated with these rights.

- Users may download and print one copy of any publication from the public portal for the purpose of private study or research.

- You may not further distribute the material or use it for any profit-making activity or commercial gain

- You may freely distribute the URL identifying the publication in the public portal 


\section{Distributed Service Restoration of Active Electrical Distribution Systems using ADMM}

\author{
Juan Camilo López and Marcos J. Rider \\ School of Electrical and Computing of Engineering \\ Department of Energy Systems \\ State University of Campinas (UNICAMP) \\ Campinas, São Paulo, Brazil \\ Email: jclopeza@fee.dsee.unicamp.br \\ mjrider@fee.dsee.unicamp.br
}

\author{
Feifan Shen and Qiuwei Wu \\ Center for Electric Power and Energy \\ Department of Electrical Engineering \\ Technical University of Denmark (DTU) \\ Kgs. Lyngby, Denmark \\ Email: fshen@elektro.dtu.dk \\ qw@elektro.dtu.dk
}

\begin{abstract}
An essential aspect that contributes to the resilience of radial electrical distribution systems (EDS) is the deployment of efficient and fast service restoration methods. In the presence of a permanent fault, the service restoration minimizes the amount of de-energized consumers by reducing the area that needs to be disconnected to isolate the faulty section of the network. To do so, the service restoration algorithm can make use of automated switching devices and dispatchable energy resources allocated throughout the network to transfer loads among feeders, considering the system's operational constraints. Depending on the size of the system and the complexity of the telecommunication infrastructure, the application of centralized restoration systems may neither be practical nor flexible. Thus, in this paper, a distributed service restoration method is proposed, using the alternating direction method of multipliers (ADMM) algorithm to solve the optimization problem. As such, the original service restoration model is distributed among the nodes and branches of the system that exchange limited information with neighboring nodes and branches. Radiality constraint is also distributed by the proposed ADMM algorithm. A 44-node test system was used to conduct case studies. Results demonstrate that the proposed method efficiently solves the service restoration in active EDS without using a centralized intelligence.

Index Terms-Alternating direction method of multipliers, electrical distribution systems, radiality constraint, service restoration.
\end{abstract}

\section{INTRODUCTION}

A keystone of the planning and operation of modern electrical distribution systems (EDSs) is the quest for flexibility and resilience [1]. Flexible EDSs incorporate and exploit the capabilities of distributed automation (DA) technologies, such as switching devices and dispatchable distributed generation (DG) resources, to optimize the grid's performance. Resilient EDSs efficiently react against fortuitous situations that threaten the continuous operation of the grid. In both objectives, either flexibility and resilience, the application of optimal service restoration methods is found to be essential [2].

Since the early nineties, multiple centralized approaches for the optimal service restoration have been proposed in the specialized literature [3], many of which consider step-bystep procedures [4]-[6], fuzzy logic [7], modern heuristics [8], graph theory [9] and mathematical programming [10][12], among others. In most of the previous works, the objective function minimizes the total unsupplied demand, while guaranteeing the electrical and topological constraints of the network. Centralized methods can be very efficient, but they require a complex and bulky communication infrastructure, and their performance is highly sensitive to the size of the EDS. More recently, some works have proposed decentralized service restoration approaches using different adaptations of multi-agent systems [13]-[16]. Despite its straightforwardness and modular characteristics, multi-agent system are not based on mathematical programming. Thus, optimality of multyagent systems in terms of minimal unsupplied demand and its feasibility is not guaranteed.

Given the previous context, this paper develops and presents a new distributed service restoration algorithm. Initially, the optimal restoration problem of active EDS is presented as a mixed-integer linear programming (MILP) problem in which the faulty section of the network is isolated while minimizing the impact of the permanent outage, i.e., the unsupplied demand after the restoration process. The decision variables are the status (open or closed) of the switching devices and the operation of the dispatchable DG units, installed throughout the network. Then, the alternating direction method of multipliers (ADMM) is used to distribute the service restoration problem among the nodes and branches of the network. Electrical constraints, such as flow and generation capacities are guaranteed by the solution and, more importantly, the radiality topology of the restored portion of the network is also considered and distributed by the proposed method.

The contributions of the proposed approach are twofold:

- A MILP model for the optimal restoration of active EDS, considering a decomposable radiality constraint.

- An adaptation of the ADMM algorithm to solve the service restoration of radial EDS in a distributed fashion.

The rest of the paper is organized as follows: Section II introduces the MILP model for the optimal restoration of active EDS considering a decomposable radiality constraint. Section III presents the adaptation of the ADMM algorithm to distribute the service restoration problem. Section IV presents the tests and results obtained using a 44-node test system. Finally, conclusions are given in Section V. 


\section{Optimal Service Restoration Model}

In this paper, the optimal service restoration model of active EDS is represented by the MILP model in (1)-(6), where $N$ is the set of nodes and $B$ is the set of switchable branches.

$$
\min \sum_{i \in N}\left[c_{i}^{\mathrm{S}} P_{i}^{\mathrm{S}}+c_{i}^{\mathrm{DG}} P_{i}^{\mathrm{DG}}+c^{\mathrm{ENS}}\left(1-z_{i}\right)\right]
$$

Subject to:

$$
\begin{array}{lr}
P_{i}^{\mathrm{S}}+\sum_{l \in B(j \rightarrow i)} P_{l}-\sum_{l \in B(i \rightarrow j)} P_{l}=P_{i}^{\mathrm{D}} z_{i}-P_{i}^{\mathrm{DG}} & \forall i \in N^{(2)} \\
\underline{P_{l}} w_{l} \leq P_{l} \leq \overline{P_{l}} w_{l} & \forall l \in B \text { (3) } \\
0 \leq P_{i}^{\mathrm{DG}} \leq \overline{P_{i}^{\mathrm{DG}}} z_{i} & \forall i \in N^{(4)} \\
0 \leq P_{i}^{\mathrm{S}} \leq \overline{P_{i}^{\mathrm{S}}} & \forall i \in N^{(5)} \\
z_{i}, w_{l} \in\{0,1\} & \forall i \in N, l \in B(6)
\end{array}
$$$$
\forall l \in B(3)
$$

The objective function in (1) minimizes the total cost of production at the different sources, i.e., the cost of energy $c_{i}^{S}$ at each source multiplied by its production $P_{i}^{\mathrm{S}}$, and the cost of each DG unit $c_{i}^{\mathrm{DG}}$ multiplied by its production $P_{i}^{\mathrm{DG}}$. The last term of the objective function penalizes the binary decision variable $z_{i}$ that represents the status of each node using a constant cost of energy not supplied (ENS) $c^{\text {ENS }}$. Thus, if a node is de-energized (i.e., if $z_{i}=0$ ), a penalty will be added; otherwise, if $z_{i}=1$, there will be no cost of ENS at node $i \in N$. Other objectives, such as number of switching actions, can be easily added or removed from the objective function, as long as convexity is not compromised.

Equality constraint in (2) represents the active power balance equation at each node. For the sake of simplicity, losses and reactive power flow are disregarded from this formulation, but they will be regarded in future works. In (2), $P_{l}$ represents the flow at branch $l \in B$, in which the initial and final nodes do not need to follow a specific numbering scheme, hence, the distinction between $B(j \rightarrow i)$ and $B(i \rightarrow j)$. Function $B(j \rightarrow i)$ fetches the branches whose final node is $i$, whereas function $B(i \rightarrow j)$ fetches the branches whose initial node is $i$. Power flows through branches are limited by (3), considering the upper $\left(\overline{P_{l}}\right)$ and lower $\left(P_{l}\right)$ limits, and the binary decision variable $w_{l}$ that determines the status of each switchable branch $l$, i.e., open or closed. Production of DG units in (4) is limited by $\overline{P_{i}^{\mathrm{DG}}}$, considering that DGs can only operate if the nodes are energized, as dictated by the status of $z_{i}$. Production limits are given by (5), assuming $\overline{P_{i}^{\mathrm{S}}}=0$ for load nodes, i.e., for $n_{i}=$ "load", in which parameter $n_{i}$ determines the type of node $i$, either "load" or "source". Finally, the binary nature of of decision variables $z_{i}$ and $w_{l}$ are given by (6).

\section{A. Decomposable Radiality Constraint}

In order to complement the service restoration model in (1)(6), radiality must be guaranteed at the energized portion of the network. To do so, a set of additional constraints given by (7)-(10) are required.

$$
\begin{array}{lr}
\beta_{i j}+\beta_{j i}=w_{l} & \forall l \in B \\
\sum_{l \in B(i \rightarrow j)} \beta_{i j}=z_{i} & \forall i \in N \\
\beta_{i j}=0 & \left.\forall i \in N\right|_{n_{i}=\text { "source" }} \\
\beta_{i j}, \beta_{j i} \in\{0,1\} & \forall l \in B
\end{array}
$$

The set of equations in (7)-(10) are called spanning tree constraints [17], and together with (2), they guarantee a final radial topology of the energized portion of the network.

A unique aspect about the overall service restoration model in (1)-(10) is that each constraint is either defined for each node or each branch of the network. Thus, this decomposable structure can be exploited by distributed optimization algorithms, such as ADMM, as explained in the following section.

\section{ADMM-BASED ALGORITHM}

The ADMM algorithm is used to decompose convex optimization problems and solve them through a two-step iterative process, in which a set of variables is optimized first $(x$ update), and then another set is optimized in the second step ( $y$-update), exchanging updated primal and dual data among both sub-problems [18]. An interesting aspect about ADMM is that it can be adapted to formulate and solve distributed instances of optimal power flows, as in [19], [20].

In this section, an adaptation of the ADMM algorithm is used to solve the service restoration problem in (1)-(10). First, the augmented Lagrangian method is applied, and the nodal $x$ update and the branch $y$-update sub-problems are introduced. Then, the updating of the dual variables and the stopping criterion are shown. Finally, the mechanism used to exchange information among nodes and branches is illustrated.

\section{A. Augmented Lagrangian}

Consider a set of auxiliary variables and equality constraints given by $P_{i j}=P_{l}, P_{j i}=P_{l}, \alpha_{i j}=\beta_{i j}, \alpha_{j i}=\beta_{j i}$; $\forall l \in B$. Thus, adding the former redundant constraints to the original problem in (1)-(10) can be done through the augmented Lagrangian method as follows:

$$
\begin{aligned}
\min \left\{\left[c_{i}^{\mathrm{S}} P_{i}^{\mathrm{S}}+c_{i}^{\mathrm{DG}} P_{i}^{\mathrm{DG}}+c^{\mathrm{ENS}}\left(1-z_{i}\right)\right]+\right. \\
+\sum_{l \in B}\left[\mu_{i j}\left(P_{i j}-P_{l}\right)+\frac{1}{2} \rho\left(P_{i j}-P_{l}\right)^{2}+\right. \\
\quad+\mu_{j i}\left(P_{j i}-P_{l}\right)+\frac{1}{2} \rho\left(P_{j i}-P_{l}\right)^{2}+ \\
+\pi_{i j}\left(\beta_{i j}-\alpha_{i j}\right)+\frac{1}{2} \rho\left(\beta_{i j}-\alpha_{i j}\right)^{2} \\
\left.\left.\quad+\pi_{j i}\left(\beta_{j i}-\alpha_{j i}\right)+\frac{1}{2} \rho\left(\beta_{j i}-\alpha_{j i}\right)^{2}\right]\right\}
\end{aligned}
$$

Subject to: (2)-(10).

Where $\mu_{i j}, \mu_{j i}, \pi_{i j}$ and $\pi_{j i}$ are the dual variables associated to the additional set of equality constraints. Thus, using the method proposed in [18], the MILP model in (11) can be decomposed in two sub-problems: nodal $x$-update and branch $y$-update, as explained in the following subsections. 


\section{B. Nodal x-update}

The $x$-update sub-problem is given by (12)-(18). Superscript $x$ and $y$ are given to the variables that are optimized at each instance of the ADMM algorithm. Thus, for the $x$-update sub-problem in (12)-(18), only the variables with superscript $x$ are optimized and the rest are considered as parameters of the MILP model.

$$
\begin{gathered}
\min \left\{\left[c_{i}^{\mathrm{S}} P_{i}^{\mathrm{S}, x}+c_{i}^{\mathrm{DG}} P_{i}^{\mathrm{DG}, x}+c^{\mathrm{ENS}}\left(1-z_{i}^{x}\right)\right]+\right. \\
+\sum_{l \in B(i \rightarrow j)}\left[\mu_{i j}^{k} P_{i j}^{x}+\frac{1}{2} \rho\left(P_{i j}^{x}-P_{l}^{y}\right)^{2}+\pi_{i j}^{k} \beta_{i j}^{x}+\right. \\
\left.+\frac{1}{2} \rho\left(\beta_{i j}^{x}-\alpha_{i j}^{y}\right)^{2}\right]+\sum_{l \in B(j \rightarrow i)}\left[\mu_{j i}^{k} P_{j i}^{x}+\frac{1}{2} \rho\left(P_{j i}^{x}-P_{l}^{y}\right)^{2}+\right. \\
\left.\left.+\pi_{j i}^{k} \beta_{j i}^{x}+\frac{1}{2} \rho\left(\beta_{j i}^{x}-\alpha_{j i}^{y}\right)^{2}\right]\right\}
\end{gathered}
$$

Subject to:

$$
\begin{array}{lr}
P_{i}^{\mathrm{S}, x}+\sum_{l \in B(j \rightarrow i)} P_{j i}^{x}-\sum_{l \in B(i \rightarrow j)} P_{i j}^{x}=P_{i}^{\mathrm{D}} z_{i}^{x}-P_{i}^{\mathrm{DG}, x} \\
& \forall i \in N \\
0 \leq P_{i}^{\mathrm{DG}, x} \leq \overline{P_{i}^{\mathrm{DG}}} z_{i}^{x} & \forall i \in N \\
0 \leq P_{i}^{\mathrm{S}, x} \leq \overline{P_{i}^{\mathrm{S}}} & \forall i \in N \\
\sum_{l \in B(i \rightarrow j)} \beta_{i j}^{x}=z_{i}^{x} & \forall i \in N \\
\beta_{i j}^{x}=0 & \left.\forall i \in N\right|_{n_{i}=" \text { source" }} \\
z_{i}^{x}, \beta_{i j}^{x}, \beta_{j i}^{x} \in\{0,1\} \quad \forall i \in N, l \in B(i \rightarrow j) \cup B(j \rightarrow i)
\end{array}
$$$$
\forall i \in N(13)
$$

Note that the $x$-update sub-problem in (12)-(18) can be solved for each node at the time. Thus, an independent instance of (12)-(18) is calculated for each node of the process. The objective function in (12) is the augmented Lagrangian of the $x$-update sub-problem, considering the equivalent auxiliary variables $P_{i j}^{x}, P_{j i}^{x}, \alpha_{i j}^{x}, \alpha_{j i}^{x}$. The superscript $k$ at the dual variables $\mu_{i j}^{k}, \mu_{j i}^{k}, \pi_{i j}^{k}, \pi_{j i}^{k}$; associated to each equivalent auxiliary variable, indicates the current iteration of the convergence process. The binary nature of $\beta$ is relaxed.

\section{Branch y-update}

Once the $x$-update process has finished, the $y$-update subproblem in (19)-(22) can be solved for each switchable branch of the EDS. In this case, only the variables with superscript $y$ are optimized and the rest are considered as parameters of the MILP model. The binary nature of $\alpha$ is relaxed.

$$
\begin{array}{r}
\min \left\{\sum _ { l \in B } \left[-\left(\mu_{i j}^{k}+\mu_{j i}^{k}\right) P_{l}^{y}+\frac{1}{2} \rho\left(P_{i j}^{x}-P_{l}^{y}\right)^{2}\right.\right. \\
+\frac{1}{2} \rho\left(P_{j i}^{x}-P_{l}^{y}\right)^{2}-\pi_{i j}^{k} \alpha_{i j}^{y}+\frac{1}{2} \rho\left(\beta_{i j}^{x}-\alpha_{i j}^{y}\right)^{2} \\
\left.\left.-\pi_{j i}^{k} \alpha_{j i}^{y}+\frac{1}{2} \rho\left(\beta_{j i}^{x}-\alpha_{j i}^{y}\right)^{2}\right]\right\}
\end{array}
$$

(a) $x$-update

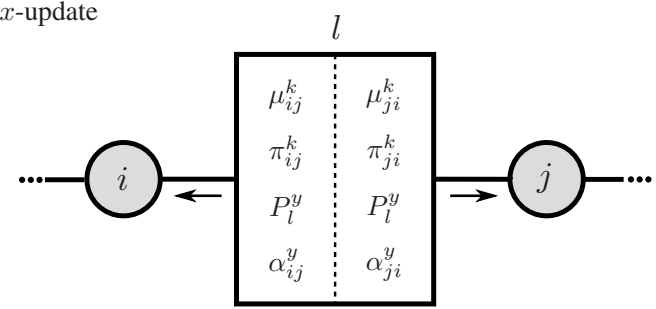

(b) $y$-update

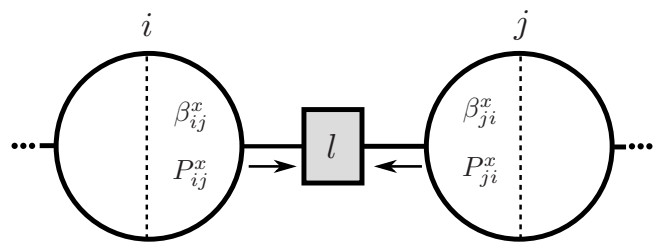

Figure 1. Exchange of information at the $x$-update and the $y$-update.

Subject to:

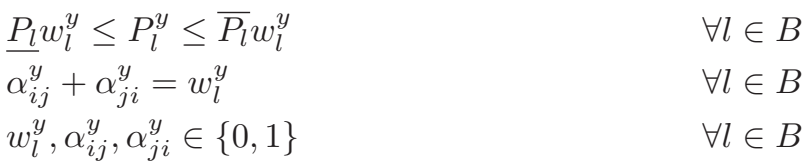

\section{Dual variables update}

Once the $y$-update process has finished, the dual variables can also be updated in the branches through (23)-(26).

$$
\begin{aligned}
\mu_{i j}^{k+1} & =\mu_{i j}^{k}+\rho\left(P_{i j}^{x}-P_{l}^{y}\right) & & \forall l \in B \\
\mu_{j i}^{k+1} & =\mu_{j i}^{k}+\rho\left(P_{j i}^{x}-P_{l}^{y}\right) & & \forall l \in B \\
\pi_{i j}^{k+1} & =\pi_{i j}^{k}+\rho\left(\beta_{i j}^{x}-\alpha_{i j}^{y}\right) & & \forall l \in B \\
\pi_{j i}^{k+1} & =\pi_{j i}^{k}+\rho\left(\beta_{j i}^{x}-\alpha_{j i}^{y}\right) & & \forall l \in B
\end{aligned}
$$$$
\forall l \in B(24)
$$

\section{E. Stopping criterion}

As shown in (27), the converge process stops when the aggregated differences between the exchanged variables is lower than a given tolerance $\epsilon$.

$$
\begin{aligned}
\sum_{l \in B}\left(\left|P_{i j}^{x}-P_{l}^{y}\right|+\right. & \left|P_{j i}^{x}-P_{l}^{y}\right| \\
& \left.+\left|\beta_{i j}^{x}-\alpha_{i j}^{y}\right|+\left|\beta_{j i}^{x}-\alpha_{j i}^{y}\right|\right) \leq \epsilon
\end{aligned}
$$

\section{F. Data exchange}

As shown in Fig. 1, during the $x$-update, the nodes are activated and they solve the nodal MILP model in (12)-(18). To do so, both nodes, $i$ and $j$, receive the primal and dual information from the switchable branch $l$ that interconnects them. Then, in the $y$-update process, the branch $l$ is activated to solve the branch MILP model in (19)-(22), after receiving the primal and dual parameters from the nodes $i$ and $j$, respectively. The exchange of information shown in Fig. 1 can be done in a distributed fashion, following the paths that communicate the nodes and the branches of the EDS. 


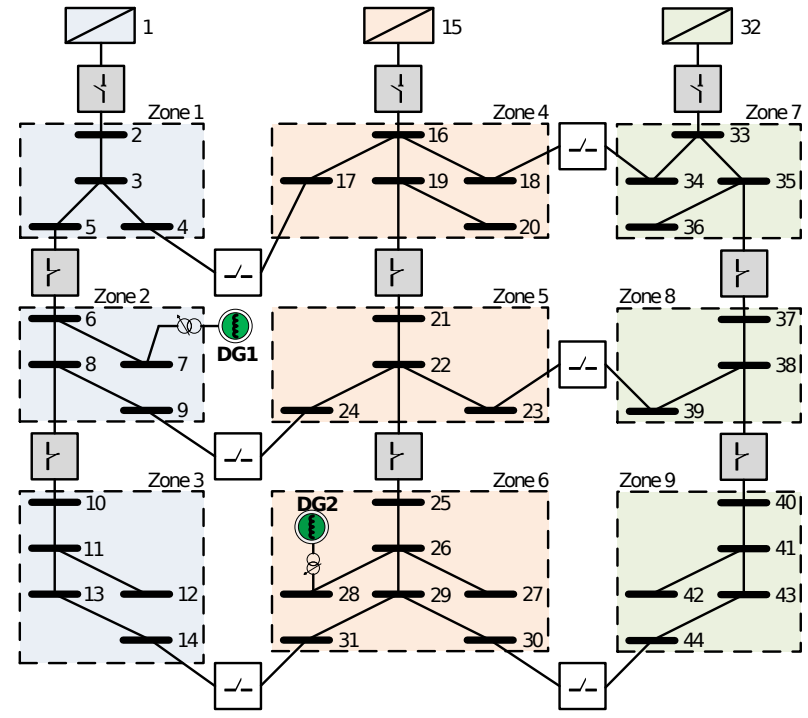

Figure 2. Initial topology of the 44-node test system

Table I

SYSTEM DATA

\begin{tabular}{|c|c|c|c|c|}
\hline Node $[i]$ & $P_{i}^{\mathrm{D}}[\mathrm{kW}]$ & $\overline{P_{i}^{\mathrm{DG}}}[\mathrm{kW}]$ & Line $[l]$ & $\overline{P_{l}}[\mathrm{~kW}]$ \\
\hline 3 & 100 & - & $1-2$ & 2000 \\
\hline 6 & 100 & - & $5-6$ & 1000 \\
\hline 7 & - & 30 & $8-10$ & 1000 \\
\hline 9 & 100 & - & $4-17$ & 1000 \\
\hline 11 & 100 & - & $9-24$ & 1000 \\
\hline 13 & 100 & - & $14-31$ & 1000 \\
\hline 16 & 100 & - & $15-16$ & 2000 \\
\hline 22 & 100 & - & $19-21$ & 1000 \\
\hline 26 & 100 & - & $22-25$ & 1000 \\
\hline 28 & - & 30 & $18-34$ & 1000 \\
\hline 29 & 100 & - & $23-39$ & 1000 \\
\hline 33 & 100 & - & $30-44$ & 1000 \\
\hline 38 & 200 & - & $32-33$ & 2000 \\
\hline 41 & 200 & - & $35-37$ & 1000 \\
\hline 43 & 200 & - & $38-40$ & 1000 \\
\hline
\end{tabular}

\section{TESTS AND RESUlts}

In order to validate the efficiency of the proposed ADMMbased algorithm, three case studies were conducted with the 44-node test system in Fig. 2. The system comprises three separated feeders: blue, red and green. There are nine zones and two DG units connected at zones 2 and 6, respectively. Open switches are represented as white-colored boxes, and closed switches are represented as gray-colored boxes. Table I shows the detailed load data (nodes with no active demands are not shown), DG units, and line flow capacities. In the service restoration model, the cost of ENS $c^{\mathrm{ENS}}$ is set to be $100 \mathrm{~m}$.u. (monetary units) to maximize the number of energized zones. The cost of energy $c_{i}^{\mathrm{S}}$ of each source node is set as $5 \mathrm{~m} . \mathrm{u} . / \mathrm{kW}$. The cost of production $c_{i}^{\mathrm{DG}}$ at each DG unit is set as $2 \mathrm{~m} . \mathrm{u} . / \mathrm{kW}$. The stopping criterion $\epsilon$ is $1 \mathrm{e}^{-4}$, and $\rho$ is 2 .

\section{A. Case 1}

In this case, the effectiveness of the proposed ADMMbased algorithm is validated. It is assumed that a permanent

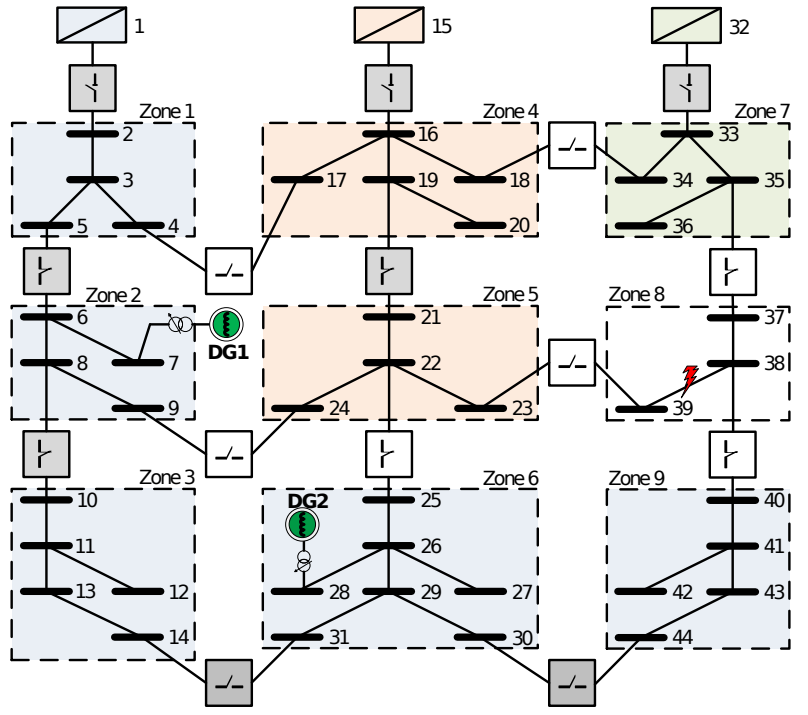

Figure 3. Restored topology of the 44-node test system

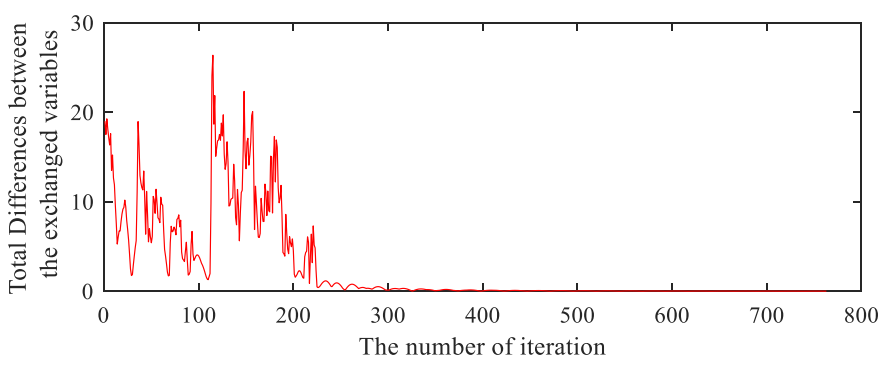

Figure 4. Iteration process in case 1

fault occurs in zone 8, and the ADMM-based algorithm is used to solve the optimal service restoration model. After convergence, the restored topology of the test system is shown in Fig. 3. Note that the faulty zone (zone 8) was isolated by opening the switch between nodes 35 and 37 and the switch bewteen nodes 38 and 40 . The switch between nodes 30 and 44 was closed to supply the load at zone 9. It is worth noting that the switch between nodes 22 and 25 was also opened and the switch between nodes 14 and 31 was closed to transfer zones 6 to the blue feeder. This happens because the minimization of the number of switching actions is not considered in the objective function. Thus, the proposed method prioritized the minimization of the unsupplied demand and the power production. The power outputs of both DG units are listed in Table II. It can be seen that both DG units use their maximum capacities because they have lower energy production costs than the main sources.

The convergence process of the ADMM-based algorithm is shown in Fig. 4 and the number of iterations is listed in Table III. It took 763 iterations to converge. As observed from the iterative curve in Fig. 4, there are oscillations during the initial stages of the convergence process, which may be caused by the presence of binary variables in each sub-problem. 
Table II

ACTIVE POWER OUTPUTS OF THE DG UNITS

\begin{tabular}{|c|c|c|}
\hline$P_{i}^{\mathrm{DG}}(\mathrm{kW})$ & DG1 & DG2 \\
\hline case1 & 30 & 30 \\
\hline case3 & 30 & 0 \\
\hline
\end{tabular}

Table III

THE NUMBERS OF ITERATIONS IN THREE CASES

\begin{tabular}{|c|c|c|c|c|c|}
\hline Case & case 1 & \multicolumn{3}{|c|}{ case2 } & case3 \\
\hline$\rho$ & 2.0 & 1.0 & 2.0 & 4.0 & 2.0 \\
\hline Iterations & 763 & 1044 & 763 & 942 & 864 \\
\hline
\end{tabular}

\section{B. Case 2}

In this case, the influence of parameter $\rho$ on the convergence of the ADMM-based algorithm is studied. Here, we conducted three simulations by using three different values for $\rho$, i.e., $\rho_{1}=1.0, \rho_{2}=2.0, \rho_{3}=4.0$. The numbers of iterations with three different $\rho$ are listed in Table III and the comparison between three iteration processes is shown in Fig. 5.

All solutions were the same as in Case 1. However, parameter $\rho$ has a big effect on the convergence of the proposed ADMM-based algorithm. Using $\rho=2$, it took the least number of iterations to reach convergence. Therefore, it is very important to choose an appropriate value for parameter $\rho$.

\section{Case 3}

In this case, we consider a large cost of production for DG unit 2 and perform the ADMM-based algorithm again. The number of iterations is listed in Table III. The power outputs of two DGs units are shown in Table II. It can been seen that the active power output of DG unit 2 became zero because it is too expensive, and DG unit 1 used his maximum output due to its low cost of production. In this case, more demands were supplied by the source nodes.

\section{CONCLUSiOnS}

This paper proposes a MILP model for the optimal restoration problem considering the decomposable radiality constraints. Then, the proposed MILP model is decomposed and distributed using an adaptation of the ADMM algorithm. The optimal solution of the MILP model is obtained by iteratively solving the nodal $x$-update sub-problem and the branch $y$ update sub-problem. Primal and dual values are exchange between neighbor nodes and branches. The simulation results demonstrate that the proposed ADMM algorithm can effectively solve the optimal restoration service problem in a distributed manner.

\section{ACKNOWLEDGMENTS}

This work was supported in part by the Brazilian institution FAPESP grant 2015/12564-1 and in part by the DTU-NTU double PhD project of the Smart City joint program.

\section{REFERENCES}

[1] D. Manz et al., "The grid of the future: Ten trends that will shape the grid over the next decade," IEEE Power and Energy Magazine, vol. 12, no. 3, pp. 26-36, May 2014.

[2] S. Harrison, "Future power system architecture," Department of Energy \& Climate Change (DECC), London, UK, Tech. Rep., 2016.

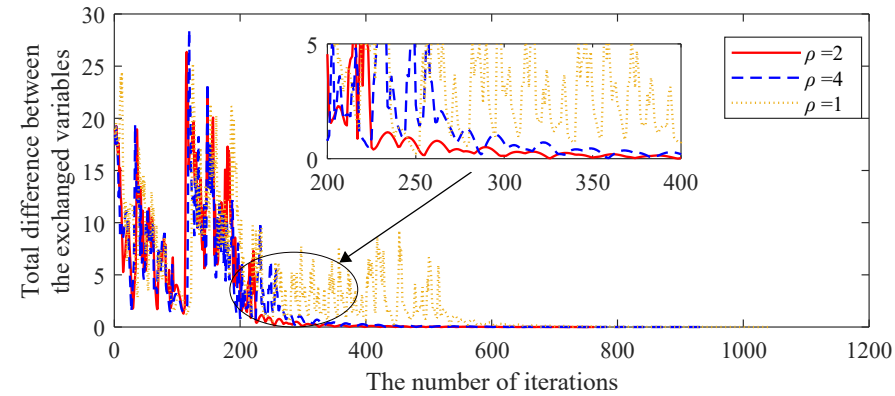

Figure 5. Iteration processes with three values for $\rho$

[3] A. Zidan et al., "Fault detection, isolation, and service restoration in distribution systems: State-of-the-art and future trends," IEEE Trans. Smart Grid, vol. 8, no. 5, pp. 2170-2185, Sept. 2017.

[4] A. L. Morelato and A. J. Monticelli, "Heuristic search approach to distribution system restoration," IEEE Trans. Power Del., vol. 4, no. 4, pp. 2235-2241, Oct. 1989.

[5] Y. Hsu et al., "Distribution system service restoration using a heuristic search approach," IEEE Trans. Power Del., vol. 7, no. 2, pp. 734-740, April 1992

[6] C. Ucak and A. Pahwa, "An analytical approach for step-by-step restoration of distribution systems following extended outages," IEEE Trans. Power Del., vol. 9, no. 3, pp. 1717-1723, July 1994.

[7] Q. Zhou, D. Shirmohammadi, and W. E. Liu, "Distribution feeder reconfiguration for service restoration and load balancing," IEEE Trans. Power Syst., vol. 12, no. 2, pp. 724-729, May 1997.

[8] S. Toune, H. Fudo, T. Genji, Y. Fukuyama, and Y. Nakanishi, "Comparative study of modern heuristic algorithms to service restoration in distribution systems," IEEE Trans. Power Del., vol. 17, no. 1, pp. 173181, Jan. 2002

[9] N. D. R. Sarma, V. C. Prasad, K. S. P. Rao, and V. Sankar, "A new network reconfiguration technique for service restoration in distribution networks," IEEE Trans. Power Del., vol. 9, no. 4, pp. 1936-1942, Oct. 1994.

[10] S. Khushalani, J. M. Solanki, and N. N. Schulz, "Optimized restoration of unbalanced distribution systems," IEEE Trans. Power Syst., vol. 22, no. 2, pp. 624-630, May 2007.

[11] P. L. Cavalcante et al., "Centralized self-healing scheme for electrical distribution systems," IEEE Trans. Smart Grid, vol. 7, no. 1, pp. 145155, Jan. 2016

[12] J. C. Lopez, J. F. Franco, M. J. Rider, and R. Romero, "Optimal restoration/maintenance switching sequence of unbalanced three-phase distribution systems," IEEE Trans. Smart Grid, 2018, to be published.

[13] T. Nagata and H. Sasaki, "A multi-agent approach to power system restoration," IEEE Trans. Power Syst., vol. 17, no. 2, pp. 457-462, May 2002.

[14] J. M. Solanki, S. Khushalani, and N. N. Schulz, "A multi-agent solution to distribution systems restoration," IEEE Trans. Power Syst., vol. 22, no. 3, pp. 1026-1034, Aug. 2007.

[15] A. Zidan and E. F. El-Saadany, "A cooperative multiagent framework for self-healing mechanisms in distribution systems," IEEE Trans. Smart Grid, vol. 3, no. 3, pp. 1525-1539, Sept. 2012.

[16] M. Eriksson, M. Armendariz, O. O. Vasilenko, A. Saleem, and L. Nordström, "Multiagent-based distribution automation solution for selfhealing grids," IEEE Transactions on Industrial Electronics, vol. 62, no. 4, pp. 2620-2628, April 2015.

[17] R. A. Jabr, R. Singh, and B. C. Pal, "Minimum loss network reconfiguration using mixed-integer convex programming," IEEE Trans. Power Syst., vol. 27, no. 2, pp. 1106-1115, May 2012.

[18] S. Boyd, N. Parikh, E. Chu, B. Peleato, and J. Eckstein, "Distributed optimization and statistical learning via the alternating direction method of multipliers," Foundations and Trends in Machine Learning, vol. 3, no. 1, pp. 1-122, Jan. 2010.

[19] Q. Peng and S. H. Low, "Distributed optimal power flow algorithm for radial networks, I: Balanced single phase case," IEEE Trans. Smart Grid, vol. 9, no. 1, pp. 111-121, Jan. 2018.

[20] Y. Wen, X. Qu, W. Li, X. Liu, and X. Ye, "Synergistic operation of electricity and natural gas networks via admm," IEEE Trans. Smart Grid, vol. 9, no. 5, pp. 4555-4565, Sept. 2018. 